\title{
Produção de Carne em Cordeiros Cruza Border Leicester com Ovelhas Corriedale e Ideal ${ }^{1}$
}

\author{
José Carlos da Silveira Osório 2,3, Nelson Manzoni de Oliveira ${ }^{3,4}$, Maria Teresa Moreira Osório ${ }^{2,3}$, \\ Rodrigo Desessard Jardim ${ }^{5}$, Marcelo Alves Pimentel ${ }^{2}$
}

\begin{abstract}
RESUMO - O estudo objetivou oferecer informações sobre a morfologia (in vivo e na carcaça), características comerciais, componentes do peso vivo, qualidade da carcaça e da carne em cordeiros não castrados cruzas de Border Leicester com ovelhas Corriedale e Ideal, criados em condições de campo nativo no Rio Grande do Sul, Brasil. Foram utilizados 24 cordeiros, 11 procedentes do cruzamento de Border x Corriedale e 13 de Border Leicester x Ideal, sacrificados com 6,5 meses de idade. Foi verificado um efeito entre os cruzamentos para a perda ao resfriamento (em kg e \%), peso e percentagem da pele, peso e percentagem do pescoço e capacidade de retenção de água. Igualmente, foi verificado que há diferença no desenvolvimento relativo dos componentes do peso vivo, composição regional da carcaça e composição tecidual da paleta entre os cordeiros Border Leicester x Corriedale e Border Leicester x Ideal e, dentro de cada genotipo verificou-se uma diferença quantitativa e qualitativa entre a paleta e a perna. As diferença quanto à qualidade da carcaça e carne não apresentam importância prática que justifique uma diferenciação do produto; porém, a comercialização deve ser com base no peso de carcaça fria e não com base no peso vivo ou carcaça quente. A paleta é mais precoce que a perna em ambos grupos genéticos.
\end{abstract}

Palavras-chave: carcaça, carne, cordeiros, cruzamento, desenvolvimento, ovinos

\section{Meat Production in Male Lambs Derived from the Crossing Between Border Leicester Rams with Corriedale and Polwarth Ewes}

\begin{abstract}
This study offers information on the morphology (in live and in carcass), commercial characteristics, components of the liveweight, quality of the carcass and meat in no castrated lambs derived from the crossing between Border Leicester rams and Corriedale and Polwarth ewes, grazing on native pasture in Rio Grande do Sul, Brazil. Twenty-four lambs slaughtered at 6.5 months of age were used, from which 11 were from Border Leicester rams x Corriedale ewes and 13 were from Border Leicester rams x Polwarth ewes. Significant effects between crosses were observed for carcass looses (in kg and \%), weight and percentage of the skin and neck, and capacity of water retention. Relative development of the components of liveweight, carcass regional composition and tissue composition of shoulder differed between crosses and, within of each genetic group, there were quantitative and qualitative differences between shoulder and leg. Differences in carcass and meat quality were not important as to indicate that products are different, however, it should be pointed out that commercialization should be based on the weight of cold carcasses, rather than liveweight or weight of hot carcasses.
\end{abstract}

Key Words: carcass, crossbreed, development, lambs, meat, sheep

\section{Introdução}

A utilização do cruzamento de ovelhas adaptadas a uma região, como é o caso das raças Corriedale e Ideal ao Rio Grande do Sul, com raças paternas especializadas para carne é uma alternativa para aumentar a eficiência dos sistemas produtivos, que necessitam manter a oferta de um produto de qualidade ao longo do ano.

Resultados obtidos por Oliveira et al. (1998) e Osório et al. (1996a), mostram que cordeiros
Corriedale e Ideal apresentam crescimento e desenvolvimento similares e que o sacrifício aos 154 dias de idade e aos 222 dias de idade propiciam uma carcaça e carne, igualmente, semelhantes e que podem ser comercializadas em uma denominação específica de qualidade. Entretanto, existem diferenças entre os cruzamentos, tanto na quantidade como qualidade da carcaça e carne, segundo a raça paterna, materna, sistema de criação e idade/peso de abate (Siqueira et al., 1984; Osório et al., 1996c; Macedo et al., 1997; Bianchi et al., 1998). Além

\footnotetext{
${ }^{1}$ Apoio da FAPERGS.

2 Professor da UFPel-FAEM-Departamento de Zootecnia, Caixa Postal 354, CEP 96019-900 Pelotas, RS. E.mail: jcosorio@ufpel.tche.br; mtosorio@ufpel.tche.br; map@ufpel.tche.br

3 Bolsista do CNPq.

${ }^{4}$ Pesquisador da EMBRAPA, CPPSUL, Bagé, RS. E-mail: manzoni@cppsul.embrapa.br

${ }^{5}$ Aluno do Curso de Doutorado em Zootecnia da FAEM, UFPEL.
} 
desses fatores, outros como o sexo (Osório et al., 1999cd, 2000a) e alimentação (Avila \& Osório, 1996) influem significativamente sobre o crescimento e desenvolvimento dos cordeiros e, consequentemente, sobre a qualidade da carcaça e carne.

As dificuldades em estudar um sistema de criação para produção de carne de qualidade, certamente, está, em grande parte, limitado ao número de animais e ao trabalho de avaliação pertinente, para considerar os fatores acima citados e suas interações, que influem sobre os produtos comercializados a partir do abate do animal. Assim, os experimentos normalmente avaliam isoladamente os fatores relevantes; como é o caso de nosso estudo, que tem como objetivo oferecer informações sobre a morfologia (in vivo e da carcaça), características de importância comercial, componentes do peso vivo, qualidade da carcaça e carne e do desenvolvimento relativo dos componentes do peso vivo, composição regional da carcaça e tecidual da paleta e da perna de cordeiros não castrados procedentes do cruzamento de carneiro Border Leicester com ovelhas Corriedale e Ideal, criados em condições extensivas de campo nativo no Rio Grande do Sul (Brasil).

\section{Material e Métodos}

Foram utilizados 24 cordeiros não castrados, desmamados aos 45 dias de idade, sendo 11 procedentes do cruzamento de Border Leicester com ovelhas Corriedale e 13 do cruzamento de Border Leicester com ovelhas Ideal, criados em condições extensivas de campo nativo, com predominância de Paspalum notatum Flügge e Axonopus affinis Chase (Gonçalves $\&$ Deiro, 1983), com uma lotação de 0,5 unidade animal por hectare, no Centro de Pesquisa de Pecuária dos Campos Sulbrasileiros - CPPSUL EMBRAPA de Bagé, RS, Brasil.

Os cordeiros nasceram entre 03 e 26 de setembro de 1998 e foram abatidos em 04 de abril de 1999, com idade de $195 \pm 6$ dias.

Após jejum de 12 horas, os cordeiros foram pesados e avaliados, registrando-se "in vivo" a conformação $(1=$ muito pobre a $5=$ excelente $)$, condição corporal $(1=$ muito pobre a $5=$ excelente $)$, comprimento corporal e da perna, perímetro torácico e altura do animal. A partir do peso vivo e do comprimento corporal foi calculada a compacidade corporal (Osório et al., 1998a).

R. Bras. Zootec., v.31, n.3, p.1469-1480, 2002 (suplemento)
A seguir, os cordeiros foram abatidos e registrados individualmente seu peso de carcaça quente e seu peso dos demais componentes do peso vivo, denominados de "quinto quarto" (cabeça, patas, pele, vísceras verdes, coração, pulmões com traquéia, gordura renal e pélvica, rins) e calculada a sua proporção em relação ao peso vivo ao sacrifício.

No Laboratório de Carcaças e Carnes do Departamento de Zootecnia da Faculdade de Agronomia Eliseu Maciel da Universidade Federal de Pelotas, as carcaças foram resfriadas em câmara fria, com ar forçado, por 18 horas, a $1{ }^{\circ} \mathrm{C}$; sendo, a seguir, pesadas e avaliadas, registrando-se a conformação $(1=$ muito pobre a $5=$ excelente), estado de engorduramento ( 1 = excessivamente magra a 5 = excessivamente gorda), comprimento interno da carcaça, comprimento da perna, largura e profundidade da perna e profundidade do peito. A partir do peso de carcaça fria e de seu comprimento, foi calculada a compacidade da carcaça (Osório et al., 1998a).

Foi realizada uma secção no músculo Longissimus dorsi, entre a $12^{\mathrm{a}}$ e $13^{\mathrm{a}}$ costelas, tomando-se a espessura de gordura de cobertura, avaliando-se a cor $(1=$ rosa claro a $5=$ vermelho escuro $)$, a textura $(1=$ muito grosseira a $5=$ muito fina) e o marmoreio $(1=$ inexistente a 5 = excessivo).

No músculo Longissimus dorsi foi tomado o $\mathrm{pH}$ a zero hora (quando do abate do cordeiro) e $\mathrm{pH}$ a 24 horas após o abate, calculando-se a queda do $\mathrm{pH}$. Nesse músculo, igualmente, após 48 horas, foi verificada a capacidade de retenção de água pelo método de pressão e a cor pelo método de Hornsey, descritos em Osório et al., (1998a).

Foi realizada a separação dos componentes regionais da carcaça (pescoço, paleta, costilhar e perna), tomando-se o peso individual do corte e calculada a proporção deste em relação ao peso de carcaça fria.

Foi calculado o rendimento verdadeiro da carcaça e o rendimento comercial da carcaça (a partir do peso vivo e dos pesos de carcaça quente e fria, respectivamente), assim como as perdas por resfriamento em $\mathrm{kg}$ e \% (diferença entre o peso de carcaça quente e o peso de carcaça fria).

$\mathrm{Na}$ paleta e na perna foi feita a separação dos componentes teciduais em osso, músculo e gordura e, desta, a separação em gordura subcutânea e gordura intermuscular e outros tecidos (gânglios, fascia, nervo). Calculou-se a proporção dos componentes teciduais da paleta e da perna em relação ao peso do respectivo corte (paleta e perna). 
A comparação entre os cruzamentos e entre a composição tecidual da paleta e da perna foi através da análise de variância dos dados pelo método dos quadrados mínimos, utilizando o procedimento de modelos lineares generalizados (GLM), do programa SAS (1985).

O desenvolvimento relativo dos componentes do peso vivo (quinto quarto $=\mathrm{Y}$ ) em relação ao peso vivo (X), dos componentes regionais (cortes $=\mathrm{Y}$ ) em relação ao peso de carcaça fria $(\mathrm{X})$ e dos componentes teciduais da paleta (osso, músculo e gordura $=\mathrm{Y}$ ) em relação ao peso da paleta $(X)$ foi determinado pela equação exponencial $\mathrm{Y}=\mathrm{aX} \mathrm{X}^{\mathrm{b}}$, transformada logaritmicamente em regressão linear (Huxley, 1932), sendo os coeficientes de alometria (b) estimados pela regressão com o auxílio do programa SAS (1985), e para verificar a hipótese $b \neq 1$ foi utilizado o teste de "t" a 5\% de probabilidade.

\section{Resultados e Discussão}

Foi verificado um efeito significativo $(\mathrm{P}<0,05)$ entre os cordeiros procedentes do cruzamento de Border Leicester com ovelhas da raça Ideal e os procedentes do cruzamento de Border Leicester com ovelhas da raça Corriedale para perda por resfriamento, em $\mathrm{kg} \mathrm{e} \%$ (Tabela 1$)$.

Observa-se (Tabela 1) que no cruzamento de Border Leicester com Corriedale estão as maiores perdas por resfriamento e que não há diferença quanto a gordura de cobertura, cujo fator, normalmente as perdas por resfriamento, estão associadas e, em maior quantidade desta, há menores perdas, visto que a gordura atua como isolante térmico. Portanto, a diferença na perda por resfriamento deve ser devido ao efeito genético do cruzamento e a quantidade e distribuição da gordura de cobertura; uma vez que esta é avaliada visualmente na característica engorduramente e foi até maior nos cruzas com Corriedale (índice 2,4, com probabilidade de $\mathrm{F}=0,06$ ).

Cabe salientar que os cruzas de Border Leicester $\mathrm{x}$ Corriedale apresentaram valores médios de perda ao resfriamento superiores aos encontrados para as raças Corriedale e Ideal (Osório et al. 1996b, 1998bc) e para essas mesmas raças, em três sistemas de criação (Osório et al. 1998de), para cruzas de Hampshire Downn, castrados e não castrados (Osório et al., 1999a) para cruzas de Ile de France x Corriedale e Corriedale puro (Siqueira \& Fernandes, 1999) e para cordeiros Ideal, F1 e F2 de Texel x Ideal (Pires et al., 1999). Entretanto, estes resultados, obtidos para perdas ao resfriamento, estão de acordo com os obtidos pelo método de capacidade de retenção de água no músculo Longissimus dorsi, onde, igualmente, os cordeiros, cruzas de Border Leicester com Corriedale, apresentam maior \% de suco expelido (Tabela 5) do que os cruzas de Border Leicester com

Tabela 1 - Médias \pm erros-padrão e probabilidade de F, para as características de produção e comercial entre cordeiros cruzas de Border Leicester com ovelhas Ideal e Corriedale

Table 1 - Means \pm error standard and probability of $F$, for the production characteristics and commercial among lambs you cross of Border Leicester with sheep Ideal and Corriedale

\begin{tabular}{lccc}
\hline $\begin{array}{l}\text { Características } \\
\text { Variables }\end{array}$ & Border x Ideal & Border x Corriedale & Pr de F \\
\hline $\begin{array}{l}\text { Peso vivo ao abate }(\mathrm{kg}) \\
\text { Slaughter live weight }(\mathrm{kg})\end{array}$ & $32,985 \pm 1,020$ & $33,263 \pm 1,108$ & 0,8688 \\
$\begin{array}{l}\text { Peso carcaça quente }(\mathrm{kg}) \\
\text { Hot carcass weight }(\mathrm{kg})\end{array}$ & $15,506 \pm 0,563$ & $15,043 \pm 0,612$ & 0,5825 \\
$\begin{array}{l}\text { Peso carcaça fria }(\mathrm{kg}) \\
\text { Cold carcass weight }(\mathrm{kg})\end{array}$ & $14,672 \pm 0,608$ & $13,760 \pm 0,661$ & 0,3206 \\
$\begin{array}{l}\text { Rendimento verdadeiro (\%) } \\
\text { Carcass yield true (\%) }\end{array}$ & $47,05 \pm 1,40$ & $45,44 \pm 1,52$ & 0,4405 \\
$\begin{array}{l}\text { Rendimento comercial (\%) } \\
\text { Carcass yield commercial }(\%)\end{array}$ & $41,51 \pm 1,52$ & 0,1765 \\
$\begin{array}{l}\text { Perda por resfriamento }(\mathrm{kg}) \\
\text { Fasting losses }(\mathrm{kg})\end{array}$ & $44,41 \pm 1,40$ & $1,282 \pm 0,124$ & 0,0142 \\
$\begin{array}{l}\text { Perda por resfriamento (\%) } \\
\text { Fasting losses (\%) } \\
\text { Gordura de cobertura }(\mathrm{cm})\end{array}$ & $0,834 \pm 0,114$ & $8,52 \pm 0,91$ & 0,0371 \\
Covering fat $(\mathrm{cm})$ & $5,78 \pm 0,84$ & $0,02 \pm 0,00$ & 0,3449 \\
\hline
\end{tabular}

R. Bras. Zootec., v.31, n.3, p.1469-1480, 2002 (suplemento) 
Ideal. Contudo, é importante salientar que a diferença em \% de suco expelido, do músculo Longissimus dorsi, não apresenta importância prática que desvalorize a carne de um ou outro cruzamento, visto que os valores estão dentro da amplitude de carnes normais (Alcalde et al., 1999), mas essa diferença apresenta sim importância comercial quantitativa.

Verifica-se que os cordeiros, cruza de Border Leicester com Corriedale, apresentaram, em relação aos cruza de Border Leicester com Ideal, um maior peso vivo $(+0,278 \mathrm{~kg})$, mas um menor peso de carcaça quente $(-0,463 \mathrm{~kg}$, correspondendo a $1,61 \%)$ e fria $(-0,912 \mathrm{~kg}$, correspondendo a $-2,90 \%)$ e uma morfologia, tanto in vivo como na carcaça, semelhantes (Tabela 2), e esses valores estão rela- cionados aos aspectos discutidos em relação as perdas por frio e a pele. Costa et al. (1999), assim como no presente estudo, não encontraram diferença na morfologia in vivo e da carcaça entre cordeiros Corriedale e Ideal.

A diferença de peso vivo $(0,278 \mathrm{~kg})$ dos cordeiros procedentes do cruzamento com Corriedale foi em função do maior peso de pele (Tabela 3) destes $(+0,712 \mathrm{~kg}$, correspondente a $+1,95 \%)$ em relação aos cordeiros procedentes do cruzamento com Ideal; sendo que a diferença devida a pele aumenta com a idade, pois tanto o peso como a proporção da pele em relação ao peso vivo variam e incrementam sua importância com a idade em cordeiros Corriedale e Ideal (Osório et al., 2000b).

Tabela 2 - Médias \pm erros-padrão e probabilidade de F, para morfologia in vivo e da carcaça, entre cordeiros cruzas de Border Leicester com ovelhas Ideal e Corriedale

Table 2 - Means \pm error standard and probability of $F$, for the morphology in lives and of the carcass, among lambs you cross of Border Leicester with sheep Ideal and Corriedale

\begin{tabular}{|c|c|c|c|}
\hline $\begin{array}{l}\text { Características } \\
\text { Variables } \\
\end{array}$ & Border x Ideal & Border x Corriedale & Pr de F \\
\hline \multicolumn{4}{|l|}{ In vivo } \\
\hline $\begin{array}{l}\text { Conformação }(1-5) \\
\text { Conformation }(1-5)\end{array}$ & $3,2 \pm 0,2$ & $3,0 \pm 0,2$ & 0,5446 \\
\hline $\begin{array}{l}\text { Condição corporal (1-5) } \\
\text { Body condition }(1-5)\end{array}$ & $2,7 \pm 0,1$ & $2,7 \pm 0,1$ & 0,9859 \\
\hline $\begin{array}{l}\text { Compacidade corporal }(\mathrm{kg} / \mathrm{cm}) \\
\text { Body compacity }(\mathrm{kg} / \mathrm{cm})\end{array}$ & $0,561 \pm 0,015$ & $0,589 \pm 0,016$ & 0,2064 \\
\hline $\begin{array}{l}\text { Comprimento corporal }(\mathrm{cm}) \\
\text { Body length }(\mathrm{cm})\end{array}$ & $58,8 \pm 0,8$ & $56,4 \pm 0,9$ & 0,0575 \\
\hline $\begin{array}{l}\text { Comprimento da perna }(\mathrm{cm}) \\
\text { Length of the leg }(\mathrm{cm})\end{array}$ & $51,6 \pm 0,5$ & $51,2 \pm 0,6$ & 0,5664 \\
\hline $\begin{array}{l}\text { Altura }(\mathrm{cm}) \\
\text { Height }(\mathrm{cm})\end{array}$ & $59,9 \pm 1,3$ & $62,9 \pm 1,4$ & 0,1382 \\
\hline $\begin{array}{l}\text { Perímetro toráxico }(\mathrm{cm}) \\
\text { Thoraxic perimeter }(\mathrm{cm})\end{array}$ & $75,8 \pm 1,0$ & $74,6 \pm 1,1$ & 0,4565 \\
\hline $\begin{array}{l}\text { Na carcaça } \\
\text { In the carcass }(\mathrm{cm})\end{array}$ & & & \\
\hline $\begin{array}{l}\text { Conformação }(1-5) \\
\text { Conformation }(1-5)\end{array}$ & $2,3 \pm 0,1$ & $2,5 \pm 0,1$ & 0,3299 \\
\hline $\begin{array}{l}\text { Engorduramento }(1-5) \\
\text { Score of fat content }(1-5)\end{array}$ & $2,0 \pm 0,1$ & $2,4 \pm 0,1$ & 0,0637 \\
\hline $\begin{array}{l}\text { Compacidade carcaça }(\mathrm{kg} / \mathrm{cm}) \\
\text { Compacity carcass }(\mathrm{kg} / \mathrm{cm})\end{array}$ & $0,258 \pm 0,009$ & $0,246 \pm 0,009$ & 0,3721 \\
\hline $\begin{array}{l}\text { Comprimento da carcaça }(\mathrm{cm}) \\
\text { Length of the carcass }(\mathrm{cm})\end{array}$ & $56,7 \pm 0,6$ & $55,9 \pm 0,7$ & 0,4256 \\
\hline $\begin{array}{l}\text { Comprimento da perna }(\mathrm{cm}) \\
\text { Length of the leg }(\mathrm{cm})\end{array}$ & $37,2 \pm 0,5$ & $37,5 \pm 0,5$ & 0,6082 \\
\hline $\begin{array}{l}\text { Profundidade do peito }(\mathrm{cm}) \\
\text { Depth of the chest }(\mathrm{cm})\end{array}$ & $23,9 \pm 0,4$ & $22,6 \pm 0,4$ & 0,0370 \\
\hline $\begin{array}{l}\text { Largura da perna }(\mathrm{cm}) \\
\text { Width of the leg }(\mathrm{cm})\end{array}$ & $9,1 \pm 0,2$ & $8,6 \pm 0,2$ & 0,1229 \\
\hline $\begin{array}{l}\text { Profundidade da perna }(\mathrm{cm}) \\
\text { Depth of the leg }(\mathrm{cm})\end{array}$ & $12,8 \pm 0,3$ & $12,4 \pm 0,3$ & 0,3666 \\
\hline
\end{tabular}

R. Bras. Zootec., v.31, n.3, p.1469-1480, 2002 (suplemento) 
Tabela 3 - Médias \pm erros-padrão e probabilidade de F, para os componentes do peso vivo, entre cordeiros cruzas de Border Leicester com ovelhas Ideal e Corriedale

Table 3 - Means \pm error standard and probability of $F$, for the components of the alive weight, among lambs cross of Border Leicester with sheep Ideal and Corriedale

\begin{tabular}{|c|c|c|c|}
\hline $\begin{array}{l}\text { Características } \\
\text { Variables }\end{array}$ & Border x Ideal & Border x Corriedale & Pr de F \\
\hline $\begin{array}{l}\text { Carcaça quente }(\mathrm{kg}) \\
\text { Hot carcass }(\mathrm{kg})\end{array}$ & $15,507 \pm 0,563$ & $15,043 \pm 0,612$ & 0,5825 \\
\hline $\begin{array}{l}\text { Cabeça }(\mathrm{kg}) \\
\text { Head }(\mathrm{kg})\end{array}$ & $1,314 \pm 0,063$ & $1,427 \pm 0,068$ & 0,2316 \\
\hline $\begin{array}{l}\text { Patas }(\mathrm{kg}) \\
\text { Paws (kg) }\end{array}$ & $0,746 \pm 0,023$ & $0,776 \pm 0,025$ & 0,3740 \\
\hline $\begin{array}{l}\text { Pele (kg) } \\
\text { Skin (kg) }\end{array}$ & $5,644 \pm 0,183$ & $6,356 \pm 0,199$ & 0,0151 \\
\hline $\begin{array}{l}\text { Vísceras verdes }(\mathrm{kg}) \\
\text { Green visceras }(\mathrm{kg})\end{array}$ & $9,071 \pm 0,316$ & $8,478 \pm 0,343$ & 0,2165 \\
\hline $\begin{array}{l}\text { Coração }(\mathrm{kg}) \\
\text { Heart }(\mathrm{kg})\end{array}$ & $0,180 \pm 0,009$ & $0,181 \pm 0,009$ & 0,9527 \\
\hline $\begin{array}{l}\text { Pulmões com traquéia }(\mathrm{kg}) \\
\text { Lungs with windpipe (kg) }\end{array}$ & $0,810 \pm 0,032$ & $0,749 \pm 0,035$ & 0,2031 \\
\hline $\begin{array}{l}\text { Fígado }(\mathrm{kg}) \\
\text { Liver }(\mathrm{kg})\end{array}$ & $0,486 \pm 0,020$ & $0,528 \pm 0,021$ & 0,1572 \\
\hline $\begin{array}{l}\text { Gordura renal e pélvica }(\mathrm{kg}) \\
\text { Renal and pelvic fat ( } \mathrm{kg} \text { ) }\end{array}$ & $0,276 \pm 0,034$ & $0,299 \pm 0,037$ & 0,6523 \\
\hline $\begin{array}{l}\text { Rins }(\mathrm{kg}) \\
\text { Kidneys (kg) }\end{array}$ & $0,089 \pm 0,002$ & $0,093 \pm 0,002$ & 0,2555 \\
\hline $\begin{array}{l}\text { Carcaça quente (\%) } \\
\text { Hot carcass }(\%)\end{array}$ & $47,05 \pm 1,40$ & $45,44 \pm 1,52$ & 0,4405 \\
\hline $\begin{array}{l}\text { Cabeça }(\%) \\
\text { Head }(\%)\end{array}$ & $4,01 \pm 0,22$ & $4,34 \pm 0,23$ & 0,3132 \\
\hline $\begin{array}{l}\text { Patas (\%) } \\
\text { Paws (\%) }\end{array}$ & $2,27 \pm 0,07$ & $2,35 \pm 0,07$ & 0,4354 \\
\hline $\begin{array}{l}\text { Pele }(\%) \\
\text { Skin }(\%)\end{array}$ & $17,28 \pm 0,65$ & $19,23 \pm 0,70$ & 0,0532 \\
\hline $\begin{array}{l}\text { Vísceras verdes (\%) } \\
\text { Green visceras (\%) }\end{array}$ & $27,58 \pm 0,94$ & $25,68 \pm 1,02$ & 0,1853 \\
\hline $\begin{array}{l}\text { Coração (\%) } \\
\text { Heart (\%) }\end{array}$ & $0,54 \pm 0,02$ & $0,55 \pm 0,02$ & 0,8727 \\
\hline $\begin{array}{l}\text { Pulmões com traquéia (\%) } \\
\text { Lungs with windpipe (\%) }\end{array}$ & $2,47 \pm 0,09$ & $2,26 \pm 0,10$ & 0,1231 \\
\hline $\begin{array}{l}\text { Fígado (\%) } \\
\text { Liver (\%) }\end{array}$ & $1,48 \pm 0,06$ & $1,60 \pm 0,07$ & 0,2006 \\
\hline $\begin{array}{l}\text { Gordura renal e pélvica (\%) } \\
\text { Renal and pelvic fat (\%) }\end{array}$ & $0,84 \pm 0,11$ & $0,90 \pm 0,11$ & 0,7214 \\
\hline $\begin{array}{l}\text { Rins (\%) } \\
\text { Kidneys (\%) }\end{array}$ & $0,27 \pm 0,01$ & $0,28 \pm 0,01$ & 0,4413 \\
\hline
\end{tabular}


Considerando que a raça Corriedale produz uma lã mais grossa, com maior comprimento de mecha e um velo de peso superior que a raça Ideal (Cardellino et al., 1984), os resultados obtidos neste estudo estão coerentes. Sendo que esta diferença para pele apresenta um importante fator de variação que é o peso vivo/maturidade do animal (Pires et al., 2000).

Os resultados (Tabelas 1, 2 e 3) mostram que a maior proporção do animal corresponde a outros componentes que não a carcaça $(52,95 \%$ e $54,56 \%$, respectivamente para os cruzas com Ideal e Corriedale) e que agregariam valor diferenciado em cada grupo genético. Portanto, a comercialização deve levar em consideração o "quinto quarto" e não somente a carcaça ou o peso vivo. Por outro lado, verifica-se que as características dos componentes do peso vivo, principalmente a pele, variam independentes da morfologia normalmente utilizada para produção de carne, sendo necessária uma avaliação da lã e/ou da pele.

A diferença no corte pescoço, em $\mathrm{kg}$ e \%, (Tabela 4), entre os cordeiros procedentes do cruzamento Border Leicester $\mathrm{x}$ Ideal e os Border Leicester x Corriedale, pode ser devido ao ato de separação dos componentes regionais da carcaça, uma vez que este corte não tem um ponto anatômico que facilite sua separação. Porém, essa diferença não apresenta importância prática que justifique uma comercialização em separado dos cordeiros cruzas de Border Leicester com Ideal ou Corriedale. Cabe salientar que o pescoço não é um corte de primeira categoria (Alcalde et al., 1999) e que são similares os resultados dos demais cortes da carcaça, entre os cordeiros Border x Ideal e os Border x Corriedale (Tabela 4).

Os resultados mostram, portanto, que as raças maternas, Corriedale e Ideal, quando cruzadas com Border Leicester, não ocasionam diferenças sobre a composição regional da carcaça e isso deve-se a essas raças apresentarem similar morfologia e seus cordeiros, até os 222 dias de idade, mostrarem um crescimento e características de carcaça semelhantes, como foi verificado por Oliveira et al. (1996), Osório et al. (1999ab) e Roque et al. (1999). Entretanto, existem fatores de variação, como o nível nutritivo, que podem modificar a velocidade de formação dos órgãos e tecidos que formam a massa do corpo, como mostram os resultados clássicos de Palson \& Verges (1952ab).

Da mesma maneira que a composição regional depende do formato do animal, a composição dos tecidos, ou seja, a proporção de músculo e gordura, depende do formato do animal, a semelhante peso de carcaça (Colomer, 1988). Os resultados da Tabela 5 ilustram e corroboram com os apresentados por Colomer (1988).

Tabela 4 - Médias \pm erros-padrão e probabilidade de F, para a composição regional e tecidual e da carne, entre cordeiros cruzas de Border Leicester com ovelhas Ideal e Corriedale

Table 4 - Means \pm error standard and probability of $F$, for the regional composition and tecidual and of the meat, among lambs cross of Border Leicester with sheep Ideal and Corriedale

\begin{tabular}{|c|c|c|c|}
\hline $\begin{array}{l}\text { Características } \\
\text { Variables }\end{array}$ & Border x Ideal & Border $\mathrm{x}$ Corriedale & Pr de F \\
\hline \multicolumn{4}{|c|}{ Composição regional } \\
\hline \multicolumn{4}{|c|}{ Regional composition } \\
\hline Pescoço (kg) & $0,650 \pm 0,039$ & $0,512 \pm 0,042$ & 0,0251 \\
\hline \multicolumn{4}{|l|}{ Neck $(\mathrm{kg})$} \\
\hline Paleta (kg) & $1,408 \pm 0,042$ & $1,415 \pm 0,046$ & 0,9139 \\
\hline \multicolumn{4}{|l|}{ Shoulder (kg) } \\
\hline Costilhar (kg) & $2,638 \pm 0,141$ & $2,399 \pm 0,161$ & 0,2745 \\
\hline \multicolumn{4}{|l|}{$R i b(\mathrm{~kg})$} \\
\hline Perna (kg) & $2,443 \pm 0,103$ & $2,379 \pm 0,112$ & 0,6790 \\
\hline \multicolumn{4}{|l|}{$\operatorname{Leg}(\mathrm{kg})$} \\
\hline Pescoço (\%) & $9,07 \pm 0,34$ & $7,92 \pm 0,38$ & 0,0345 \\
\hline \multicolumn{4}{|l|}{ Neck (\%) } \\
\hline Paleta $(\%)$ & $19,96 \pm 0,39$ & $21,13 \pm 0,45$ & 0,0616 \\
\hline \multicolumn{4}{|l|}{ Shoulder (\%) } \\
\hline Costilhar(\%) & $36,68 \pm 0,69$ & $35,39 \pm 0,78$ & 0,2278 \\
\hline \multicolumn{4}{|l|}{ Rib (\%) } \\
\hline Perna (\%) & $34,29 \pm 0,45$ & $35,56 \pm 0,52$ & 0,0780 \\
\hline $\operatorname{Leg}(\%)$ & & & \\
\hline
\end{tabular}

R. Bras. Zootec., v.31, n.3, p.1469-1480, 2002 (suplemento) 
Tabela 5 - Médias \pm erros-padrão e probabilidade de F, para a composição tecidual e da carne, entre cordeiros cruzas de Border Leicester com ovelhas Ideal e Corriedale

Table 5 - Means \pm error standard and probability of $F$, for the tecidual composition and of the meat, among lambs you cross of Border Leicester with sheep Ideal and Corriedale

\begin{tabular}{|c|c|c|c|}
\hline $\begin{array}{l}\text { Características } \\
\text { Variables }\end{array}$ & Border x Ideal & Border $x$ Corriedale & Pr de F \\
\hline \multicolumn{4}{|l|}{ Composição tecidual } \\
\hline \multicolumn{4}{|l|}{ Composition tecidual } \\
\hline Paleta: osso (kg) & $0,308 \pm 0,011$ & $0,320 \pm 0,012$ & 0,4565 \\
\hline \multicolumn{4}{|l|}{ Shoulder: bone (kg) } \\
\hline Músculo (kg) & $0,689 \pm 0,027$ & $0,681 \pm 0,028$ & 0,8327 \\
\hline \multicolumn{4}{|l|}{ Muscle (kg) } \\
\hline Gordura (kg) & $0,308 \pm 0,016$ & $0,317 \pm 0,016$ & 0,6678 \\
\hline \multicolumn{4}{|l|}{ Fat $(\mathrm{kg})$} \\
\hline G. subcutânea (kg) & $0,159 \pm 0,018$ & $0,157 \pm 0,019$ & 0,9375 \\
\hline \multicolumn{4}{|l|}{ G. subcutaneous (kg) } \\
\hline G. intermuscular (kg) & $0,149 \pm 0,013$ & $0,160 \pm 0,013$ & 0,5410 \\
\hline \multicolumn{4}{|l|}{ G. intermuscular (kg) } \\
\hline Outros $(\mathrm{kg})$ & $0,058 \pm 0,006$ & $0,054 \pm 0,006$ & 0,6401 \\
\hline \multicolumn{4}{|l|}{ Other (kg) } \\
\hline Osso (\%) & $22,08 \pm 0,52$ & $22,63 \pm 0,55$ & 0,4762 \\
\hline \multicolumn{4}{|l|}{ Bone (\%) } \\
\hline Músculo (\%) & $49,02 \pm 0,76$ & $48,15 \pm 0,80$ & 0,4415 \\
\hline \multicolumn{4}{|l|}{ Muscle (\%) } \\
\hline Gordura (\%) & $21,99 \pm 0,80$ & $22,36 \pm 0,84$ & 0,7503 \\
\hline \multicolumn{4}{|l|}{ Fat $(\%)$} \\
\hline \multicolumn{4}{|l|}{$\begin{array}{l}\text { Carne } \\
\text { Meat }\end{array}$} \\
\hline \multicolumn{4}{|l|}{ Meat } \\
\hline Cor (índice de 1 a 5 ) & $2,8 \pm 0,1$ & $2,7 \pm 0,1$ & 0,5656 \\
\hline \multicolumn{4}{|l|}{ Color (index from 1 to 5 ) } \\
\hline Textura (índice de 1 a 5) & $2,2 \pm 0,1$ & $2,3 \pm 0,1$ & 0,8317 \\
\hline \multicolumn{4}{|l|}{ Texture (index from 1 to 5 ) } \\
\hline Marmoreio (índice de 1 a 5) & $2,2 \pm 0,1$ & $2,1 \pm 0,1$ & 0,3816 \\
\hline \multicolumn{4}{|l|}{ I marble (index from 1 to 5 ) } \\
\hline pH zero hora (Longissimus dorsi) & $6,7 \pm 0,1$ & $6,6 \pm 0,1$ & 0,4093 \\
\hline \multicolumn{4}{|l|}{ pH zero hour (Longissimus dorsi) } \\
\hline \multicolumn{4}{|l|}{$\begin{array}{l}\text { pH } 24 \text { horas (Longissimus dorsi) } \\
\text { pH } 24 \text { hour (Longissimus dorsi) }\end{array}$} \\
\hline Queda $\mathrm{pH}$ & $0,8 \pm 0,1$ & $0,9 \pm 0,1$ & 0,7214 \\
\hline \multicolumn{4}{|l|}{ Fall $p H$} \\
\hline Queda pH (\%) & $12,73 \pm 0,93$ & $11,43 \pm 1,01$ & 0,3500 \\
\hline \multicolumn{4}{|l|}{ Fall $p H(\%)$} \\
\hline CRA (\%suco expelido) & $17,68 \pm 0,61$ & $19,62 \pm 0,67$ & 0,0436 \\
\hline CRA (expelled\% \%uice) & & & \\
\hline Cor(Fehemínico/grama) & $52,4 \pm 1,3$ & $50,8 \pm 1,4$ & 0,4024 \\
\hline Color (Faith heminico/grama) & & & \\
\hline
\end{tabular}

CRA = capacidade de retenção de água (CRA = capacity of retention of water). 
Os cordeiros procedentes do cruzamento de Border Leicester com ovelhas Corriedale ou Ideal apresentam uma composição tecidual semelhante e uma carne com características igualmente similares; exceção para a característica de capacidade de retenção de água, anteriormente discutida, juntamente com as perdas por frio, cujos valores encontramse entre a amplitude de carnes normais de qualidade.

O desenvolvimento relativo dos componentes do peso vivo (Tabela 6) e dos componentes regionais da carcaça e dos teciduais da paleta (Tabela 7) mostraram que houve pequenas diferença; os valores, entretanto, não foram antagônicos, nem incoerentes, entre os cordeiros procedentes do cruzamento de Border Leicester x Corriedale e os de Border Leicester x Ideal.

Os resultados (Tabela 6) mostraram que o desenvolvimento da carcaça em relação ao desenvolvimento do peso vivo, dentro das amplitudes de peso, não apresentaram igual significância estatística $(b \neq 1)$ em função da variabilidade (sb) entre os cordeiros

Tabela 6 - Coeficientes de alometria (b), erro padrão $\left(s^{b}\right)$ e coeficientes de determinação $\left(R^{2}\right)$ dos componentes do peso vivo

Table 6 - Alometria coefficients (b), error stander (sb) and determination coefficients $\left(R^{2}\right)$ of the components of the liveweight

\begin{tabular}{|c|c|c|c|c|}
\hline $\begin{array}{l}\text { Características } \\
\text { Characteristics }\end{array}$ & $\begin{array}{l}\text { Grupo genético } \\
\text { Genetic groups }\end{array}$ & $\mathrm{b} \pm \mathrm{s}$ & $\mathrm{b} \neq 1$ & $\mathrm{R}^{2}(\%)$ \\
\hline Carcaça & Border x Ideal & $0,932 \pm 0,260$ & n.s. & 56,25 \\
\hline \multirow[t]{2}{*}{ Carcass } & Border x Corriedale & $0,459 \pm 0,190$ & $*$ & 49,35 \\
\hline & Geral & $0,779 \pm 0,188$ & n.s. & 48,73 \\
\hline Cabeça & Border x Ideal & $0,537 \pm 0,163$ & $*$ & 52,08 \\
\hline \multirow[t]{2}{*}{ Head } & Border x Corriedale & $0,310 \pm 0,291$ & $*$ & 15,88 \\
\hline & Geral & $0,482 \pm 0,136$ & $*$ & 40,97 \\
\hline Patas & Border x Ideal & $0,648 \pm 0,147$ & $*$ & 65,97 \\
\hline \multirow{2}{*}{ Paws } & Border x Corriedale & $0,561 \pm 0,462$ & n.s. & 19,74 \\
\hline & Geral & $0,627 \pm 0,165$ & $*$ & 44,61 \\
\hline Pele & Border x Ideal & $0,315 \pm 0,193$ & $*$ & 20,94 \\
\hline \multirow{2}{*}{ Skin } & Border x Corriedale & $0,449 \pm 0,495$ & n.s. & 12,06 \\
\hline & Geral & $0,391 \pm 0,205$ & $*$ & 16,82 \\
\hline Vísceras brancas & Border x Ideal & $0,875 \pm 0,292$ & n.s. & 47,37 \\
\hline \multirow[t]{2}{*}{ White visceras } & Border x Corriedale & $0,490 \pm 0,267$ & n.s. & 35,96 \\
\hline & Geral & $0,721 \pm 0,227$ & n.s. & 35,86 \\
\hline Coração & Border x Ideal & $1,004 \pm 0,218$ & n.s. & 68,02 \\
\hline \multirow{2}{*}{ Heart } & Border x Corriedale & $0,262 \pm 0,717$ & n.s. & 02,17 \\
\hline & Geral & $0,795 \pm 0,264$ & n.s. & 33,50 \\
\hline Pulmões & Border x Ideal & $0,590 \pm 0,259$ & n.s. & 34,07 \\
\hline \multirow[t]{2}{*}{ Lungs } & Border x Corriedale & $0,999 \pm 0,499$ & n.s. & 39,99 \\
\hline & Geral & $0,661 \pm 0,233$ & n.s. & 30,82 \\
\hline Fígado & Border x Ideal & $0,607 \pm 0,213$ & n.s. & 44,80 \\
\hline \multirow[t]{2}{*}{ Liver } & Border x Corriedale & $0,148 \pm 0,504$ & n.s. & 01,41 \\
\hline & Geral & $0,494 \pm 0,206$ & $*$ & 24,19 \\
\hline Gordura interna & Border x Ideal & $1,145 \pm 1,066$ & n.s. & 10,35 \\
\hline \multirow{2}{*}{ Fat interns } & Border x Corriedale & $0,317 \pm 1,788$ & n.s. & 00,52 \\
\hline & Geral & $0,841 \pm 0,875$ & n.s. & 04,88 \\
\hline Testículos & Border x Ideal & $1,199 \pm 0,917$ & n.s. & 14,59 \\
\hline \multirow[t]{2}{*}{ Testicles } & Border x Corriedale & $1,359 \pm 1,148$ & n.s. & 18,93 \\
\hline & Geral & $1,198 \pm 0,677$ & n.s. & 14,82 \\
\hline Pênis & Border x Ideal & $0,008 \pm 0,728$ & n.s. & 00,00 \\
\hline \multirow[t]{2}{*}{ Penises } & Border x Corriedale & $0,087 \pm 0,969$ & n.s. & 00,13 \\
\hline & Geral & $0,029 \pm 0,543$ & n.s. & 00,02 \\
\hline Bexiga & Border x Ideal & $0,226 \pm 0,978$ & n.s. & 00,53 \\
\hline \multirow{2}{*}{ Bladder } & Border x Corriedale & $0,621 \pm 2,075$ & n.s. & 01,47 \\
\hline & Geral & $0,183 \pm 0,910$ & n.s. & 00,22 \\
\hline Rins & Border x Ideal & $0,490 \pm 0,155$ & $*$ & 49,90 \\
\hline \multirow[t]{2}{*}{ Kidneys } & Border x Corriedale & $0,156 \pm 0,353$ & $*$ & 03,16 \\
\hline & Geral & $0,409 \pm 0,147$ & $*$ & 29,97 \\
\hline Gordura renal & Border x Ideal & $0,680 \pm 1,276$ & n.s. & 02,77 \\
\hline \multirow[t]{2}{*}{ Renalfat } & Border x Corriedale & $0,029 \pm 1,971$ & n.s. & 00,00 \\
\hline & Geral & $0,557 \pm 1,001$ & n.s. & 01,69 \\
\hline
\end{tabular}

R. Bras. Zootec., v.31, n.3, p.1469-1480, 2002 (suplemento) 
Tabela 7 - Coeficientes de alometria (b), erro padrão $\left(s^{b}\right)$ e coeficientes de determinação $\left(R^{2}\right)$ dos componentes regionais da carcaça e teciduais da paleta

Table 7 - Alometria coefficients (b), error stander ( $(s b)$ and determination coefficients (R2) of the regional components of the carcass and teciduais of the shoulder

\begin{tabular}{|c|c|c|c|c|}
\hline $\begin{array}{l}\text { Características } \\
\text { Characteristics }\end{array}$ & $\begin{array}{l}\text { Grupo genético } \\
\text { Genetics groups }\end{array}$ & $\mathrm{b} \pm \mathrm{s}^{\mathrm{b}}$ & $b \neq 1$ & $\mathrm{R}^{2}(\%)$ \\
\hline Pescoço & Border x Ideal & $1,072 \pm 0,216$ & n.s. & 69,02 \\
\hline \multirow[t]{2}{*}{ Neck } & Border x Corriedale & $0,245 \pm 0,486$ & n.s. & 03,07 \\
\hline & Geral & $0,996 \pm 0,220$ & n.s. & 49,34 \\
\hline Paleta & Border x Ideal & $0,669 \pm 0,059$ & $*$ & 92,02 \\
\hline \multirow[t]{2}{*}{ Shoulder } & Border x Corriedale & $0,610 \pm 0,059$ & $*$ & 55,37 \\
\hline & Geral & $0,642 \pm 0,069$ & $*$ & 80,54 \\
\hline Costilhar & Border x Ideal & $1,235 \pm 0,087$ & $*$ & 94,86 \\
\hline \multirow{2}{*}{ Rib } & Border x Corriedale & $1,258 \pm 0,206$ & n.s. & 82,34 \\
\hline & Geral & $1,250 \pm 0,079$ & $*$ & 92,33 \\
\hline Perna & Border x Ideal & $0,955 \pm 0,082$ & n.s. & 92,57 \\
\hline \multirow[t]{2}{*}{ Leg } & Border x Corriedale & $1,068 \pm 0,152$ & n.s. & 85,98 \\
\hline & Geral & $0,959 \pm 0,070$ & n.s. & 89,83 \\
\hline Osso da paleta & Border x Ideal & $0,810 \pm 0,179$ & n.s. & 67,24 \\
\hline \multirow[t]{2}{*}{ Bone of the shoulder } & Border x Corriedale & $1,100 \pm 0,323$ & n.s. & 56,32 \\
\hline & Geral & $0,886 \pm 0,153$ & n.s. & 61,56 \\
\hline Músculo da Paleta & Border x Ideal & $1,221 \pm 0,081$ & $*$ & 95,75 \\
\hline \multirow[t]{2}{*}{ Muscle of the shoulder } & Border x Corriedale & $0,812 \pm 0,254$ & n.s. & 53,12 \\
\hline & Geral & $1,119 \pm 0,103$ & n.s. & 84,88 \\
\hline Gordura da paleta & Border x Ideal & $0,942 \pm 0,282$ & n.s. & 52,79 \\
\hline \multirow[t]{2}{*}{ Fat of the shoulder } & Border x Corriedale & $1,631 \pm 0,534$ & n.s. & 50,89 \\
\hline & Geral & $1,107 \pm 0,247$ & n.s. & 48,77 \\
\hline
\end{tabular}

cruzas com Ideal e Corriedale. Estes últimos apresentaram um desenvolvimento da carcaça heterogônico negativo $(b=0,459)$, mais precoces, e os cruzas com Ideal um desenvolvimento da carcaça isogônico $(b=0,932)$. Verificou-se que os cruzas com Corriedale apresentaram menor variabilidade no coeficiente de alometria da carcaça e maior para os demais componentes do peso vivo (Tabela 6). A magnitude dos valores destes coeficientes foram similares aos obtidos por Roque et al. (1999).

O costilhar, corte de menor valor comercial, apresentou um desenvolvimento tardio (Tabela 7), sendo negativo o abate a maiores pesos já que a proporção das peças de menores valores aumentam na carcaça. Para a raça Ideal, Osório et al. (2000b) verificaram que aos 222 dias de idade os cordeiros apresentaram uma proporção maior de costilhar e uma menor proporção de perna do que os cordeiros com 154 dias de idade; concordando com o desenvolvimento apresentado no presente estudo.

A paleta apresenta um desenvolvimento heterogônico precoce, e a perna, isogônico, corroborando os resultados obtidos nas Tabelas 8 e 9, nas quais se verifica, pela proporção de gordura na paleta e na perna, que a paleta foi mais precoce que a perna em ambos os grupos genéticos.
A perna apresentou maior peso e valores absolutos de osso, músculo e gordura do que a paleta em ambos os grupos genéticos (Tabelas 8 e 9 ). Porém, em valores relativos, verificou-se maior $(\mathrm{P}<0,0001)$ proporção de músculo na perna $(55,76 \%)$ do que na paleta $(50,21 \%)$ e menor $(\mathrm{P}<0,0001)$ proporção de gordura na perna $(16,44 \%)$ do que na paleta $(21,89 \%)$, nos cordeiros procedentes do cruzamento de Border Leicester com Ideal (Tabela 8).

Para os cordeiros Border Leicester x Corriedale (Tabela 9), as percentagens de músculo e osso da paleta e da perna não diferiram; a paleta, entretanto, apresentou $22,07 \%$ de gordura e a perna $16,32 \%$, diferença altamente significativa $(\mathrm{P}=0,0051)$. Porém, a percentagem de gordura na paleta foi semelhante entre os cordeiros cruzas de Border $\mathrm{x}$ Corriedale e os de Border x Ideal (Tabela 5). Para as raças Corriedale e Ideal, Osório et al. (2000b), encontraram valores percentuais semelhantes de gordura na paleta e perna, tanto aos 154 como aos 222 dias de idade dos cordeiros.

Em ambos os grupos genéticos, os cordeiros, do presente estudo, apresentaram uma paleta mais precoce que a perna (Tabelas 8 e 9). 
Tabela 8 - Médias \pm erros-padrão e probabilidade de $\mathrm{F}$, para cordeiros cruzas de Border Leicester com Ideal, entre a composição tecidual da paleta e da perna

Table 8 - Means \pm error standard and probability of $F$, for lambs you cross of Border Leicester with Ideal, among the composition tecidual of the shoulder and of the leg

\begin{tabular}{|c|c|c|c|}
\hline $\begin{array}{l}\text { Características } \\
\text { Characteristics }\end{array}$ & $\begin{array}{c}\text { Paleta } \\
\text { Shoulder }\end{array}$ & $\begin{array}{c}\text { Perna } \\
\text { Leg }\end{array}$ & PrdeF \\
\hline $\begin{array}{l}\text { Corte (gr) } \\
\text { Cut }(g r)\end{array}$ & $1400,86 \pm 179,55$ & $2414,16 \pm 453,77$ & 0,0001 \\
\hline $\begin{array}{l}\text { Músculo (gr) } \\
\text { Muscle (gr) }\end{array}$ & $704,57 \pm 109,86$ & $1347,57 \pm 272,01$ & 0,0001 \\
\hline $\begin{array}{l}\text { Osso (gr) } \\
\text { Bone (gr) }\end{array}$ & $310,06 \pm 38,71$ & $505,50 \pm 118,28$ & 0,0001 \\
\hline $\begin{array}{l}\text { Gordura(gr) } \\
\text { Fat }(g r)\end{array}$ & $307,24 \pm 56,42$ & $400,23 \pm 108,45$ & 0,0113 \\
\hline $\begin{array}{l}\text { Gordura } \\
\text { subcutânea (gr) } \\
\text { Subcutaneous } \\
\text { fat (gr) }\end{array}$ & $163,59 \pm 66,88$ & $201,92 \pm 88,63$ & 0,2253 \\
\hline $\begin{array}{l}\text { Gordurainter } \\
\text { muscular(gr) } \\
\text { Fatinter } \\
\text { muscular (gr) }\end{array}$ & $143,65 \pm 51,25$ & $198,31 \pm 47,58$ & 0,0095 \\
\hline $\begin{array}{l}\text { Gordurainter } \\
\text { muscular(gr) } \\
\text { Fatinter } \\
\text { muscular (gr) }\end{array}$ & $143,65 \pm 51,25$ & $198,31 \pm 47,58$ & 0,0095 \\
\hline $\begin{array}{l}\text { Outros (gr) } \\
\text { Other (gr) }\end{array}$ & $57,22 \pm 24,02$ & $90,33 \pm 41,68$ & 0,0205 \\
\hline $\begin{array}{l}\text { Corte }(\%) \\
\text { Cut }(\%)\end{array}$ & $19,96 \pm 1,52$ & $34,29 \pm 1,80$ & 0,0001 \\
\hline $\begin{array}{l}\text { Músculo (\%) } \\
\text { Muscle (\%) }\end{array}$ & $50,21 \pm 3,38$ & $55,76 \pm 2,70$ & 0,0001 \\
\hline $\begin{array}{l}\text { Osso (\%) } \\
\text { Bone (\%) }\end{array}$ & $22,22 \pm 1,90$ & $21,20 \pm 3,93$ & 0,4099 \\
\hline $\begin{array}{l}\text { Gordura(\%) } \\
\text { Fat (\%) }\end{array}$ & $21,89 \pm 2,55$ & $16,44 \pm 2,44$ & 0,0001 \\
\hline $\begin{array}{l}\text { Gordura } \\
\text { subcutânea (\%) }\end{array}$ & $11,64 \pm 4,72$ & $8,16 \pm 2,56$ & 0,0284 \\
\hline $\begin{array}{l}\text { Subcutaneous } \\
\text { fat (\%) }\end{array}$ & & & \\
\hline $\begin{array}{l}\text { Gordurainter } \\
\text { muscular(\%) } \\
\text { Fatinter } \\
\text { muscular (\%) }\end{array}$ & $10,25 \pm 3,67$ & $8,28 \pm 1,91$ & 0,0985 \\
\hline $\begin{array}{l}\text { Outros }(\%) \\
\text { Other }(\%)\end{array}$ & $4,15 \pm 1,73$ & $3,76 \pm 1,67$ & 0,5635 \\
\hline
\end{tabular}

\section{Conclusões}

A comercialização do animal como um todo deve levar em consideração a proporção de seus componentes e a valorização destes.

A paleta é mais precoce do que a perna.

O desenvolvimento relativo dos componentes do
Tabela 9 - Médias \pm erros-padrão e probabilidade de $F$, para cordeiros cruzas de Border Leicester com Corriedale, entre a composição tecidual da paleta e da perna

Table 9 - Means \pm error standard and probability of $F$, for lambs you cross of Border Leicester with Corriedale, among the composition tecidual of the palette and of the leg

\begin{tabular}{|c|c|c|c|}
\hline $\begin{array}{l}\text { Características } \\
\text { Characteristics }\end{array}$ & $\begin{array}{c}\text { Paleta } \\
\text { Shoulder }\end{array}$ & $\begin{array}{c}\text { Perna } \\
\text { Leg }\end{array}$ & PrdeF \\
\hline Corte(gr) & $1432,85 \pm 175,66$ & $2360,93 \pm 309,19$ & 0,0001 \\
\hline $\begin{array}{l}\text { Músculo (gr) } \\
\text { Muscle (gr) }\end{array}$ & $680,56 \pm 59,30$ & $1209.70 \pm 156,53$ & 0,0001 \\
\hline $\begin{array}{l}\text { Osso (gr) } \\
\text { Bone (gr) }\end{array}$ & $320,32 \pm 38,25$ & $492,65 \pm 155,33$ & 0,0019 \\
\hline $\begin{array}{l}\text { Gordura(gr) } \\
\text { Fat (gr) }\end{array}$ & $317,20 \pm 53,96$ & $394,17 \pm 144,01$ & 0,1125 \\
\hline $\begin{array}{l}\text { Gordura } \\
\text { subcutânea (gr) } \\
\text { Subcutaneous } \\
\text { fat (gr) }\end{array}$ & $157,09 \pm 61,02$ & $185,92 \pm 100,30$ & 0,4250 \\
\hline $\begin{array}{l}\text { Gordura } \\
\text { intermuscular(gr) } \\
\text { Fatinter } \\
\text { muscular (gr) }\end{array}$ & $160,10 \pm 37,38$ & $208,25 \pm 90,19$ & 0,1176 \\
\hline $\begin{array}{l}\text { Outros (gr) } \\
\text { Other (gr) }\end{array}$ & $53,64 \pm 15,76$ & $101,98 \pm 46,45$ & 0,0038 \\
\hline $\begin{array}{l}\text { Corte }(\%) \\
\text { Cut }(\%)\end{array}$ & $21,13 \pm 1,25$ & $35,56 \pm 1,36$ & 0,0001 \\
\hline $\begin{array}{l}\text { Músculo (\%) } \\
\text { Muscle (\%) }\end{array}$ & $48,09 \pm 6,25$ & $51,36 \pm 3,82$ & 0,1543 \\
\hline $\begin{array}{l}\text { Osso (\%) } \\
\text { Bone (\%) }\end{array}$ & $22,54 \pm 2,91$ & $20,51 \pm 6,20$ & 0,3358 \\
\hline $\begin{array}{l}\text { Gordura(\%) } \\
\text { Fat (\%) }\end{array}$ & $22,07 \pm 2,27$ & $16,32 \pm 5,62$ & 0,0051 \\
\hline $\begin{array}{l}\text { Gordura } \\
\text { subcutânea (\%) }\end{array}$ & $10,78 \pm 3,65$ & $7,55 \pm 3,70$ & 0,0526 \\
\hline $\begin{array}{l}\text { Subcutaneous } \\
\text { fat }(\%)\end{array}$ & & & \\
\hline $\begin{array}{l}\text { Gordura } \\
\text { intermuscular(\%) } \\
\text { Fatinter } \\
\text { muscular (\%) }\end{array}$ & $11,29 \pm 2,82$ & $8,77 \pm 4,05$ & 0,1055 \\
\hline $\begin{array}{l}\text { Outros }(\%) \\
\text { Other }(\%)\end{array}$ & $3,80 \pm 1,25$ & $4,28 \pm 2,01$ & 0,5163 \\
\hline
\end{tabular}

peso vivo, composição regional e tecidual, dos cordeiros cruzas de Border Leicester com Corriedale e Ideal, são semelhantes.

A qualidade da carcaça e da carne de cordeiros procedentes do cruzamento de Border Leicester com ovelhas Corriedale e Ideal é semelhante, permitindo que estas sejam comercializadas em uma mesma 
categoria ou que façam parte de uma mesma denominação específica de qualidade.

O peso vivo e o peso de carcaça quente não são os melhores parâmetros em uma comercialização que tenha como princípio valorizar a qualidade total do animal; o peso de carcaça fria é o mais recomendado.

\section{Literatura Citada}

ALCALDE, M.J.; SAÑUDO, C.; OSÓRIO, J.C. et al. Evaluación de la calidad de la canal y de la carne en canales ovinas ligeras del tipo comercial ternasco. Información Técnica Económica Agraria, v.95, n.1, p.49-64, 1999.

AVILA, V.S.; OSÓRIO, J.C.S. Efeito do sistema de criação, época de nascimento e ano na velocidade de crescimento de cordeiros. Revista Brasileira de Zootecnia, v.25, n.5, p.1007-1016, 1996.

BIANCHI, G.; GARIBOTO, G.; OLIVEIRA, G. et al. Evaluación de razas carniceras y laneras para la producción de corderos. II Jornada del proyecto producción de carne ovina en base a cruzamiento. Universidad de la República. Facultad de Agronomia. Estación Experimental Dr. Mario A. Cassinoni. Paysandú, Uruguay, 1998. 21p.

CARDELLINO, R.A.; GUERREIRO, J.L.V.; OSÓRIO, J.C.S. et al. Resultados de cinco anos do programa de melhoramento genético dos ovinos - PROMOVI: 1977-1981. EMBRAPA-UEPAE-Pelotas, 1984. 14p. (Comunicado Técnico, 18)

COLOMER, F. Estudio de los parametros que definen los caracteres cuantitativos y cualitativos de las canales bovinas. In: CURSO INTERNACIONAL SOBRE PRODUCCIÓN DE CARNE Y LECHE COM BASES EN PASTOS Y FORRAJES, 4., 1988, La Coruña, España. Anais... La Coruña:SIA, 1988. 108p.

COSTA, J.C.; OSÓRIO, J.C.; SILVA, C.A. et al. Estudo da morfologia e características comerciais em cordeiros não castrados de quatro raças. Revista Científica Rural, v.4, n.2, p.105-112, 1999.

GONÇALVES, J.O.N.; DEIRO, A.M.G. Estudo de métodos para avaliação das características estruturais do campo natural do RS. Relatório Técnico Anual 1981/1982, EMBRAPA/UEPAE, Bagé, RS, 1983. p.45-48.

HUXLEY, J.S. Problems of relative growth. London: Methuem. 1932.

MACEDO, F.A.; MARTINS, E.N.; SIQUEIRA, E.R. et al. Cruzamentos e sistemas de terminação na produção de carcaças de cordeiros. In: REUNIÃO ANUAL DA SOCIEDADE BRASILEIRA DE ZOOTECNIA, 34., 1997, Juiz de Fora. Anais... Juiz de Fora: Sociedade Brasileira de Zootecnia, 1997. p.388-390.

OLIVEIRA, N.M.; OSÓRIO, J.C.; MONTEIRO, E.M. Produção de carne em ovinos de cinco genótipos. 1. Crescimento e desenvolvimento. Ciência Rural, v.26, n.3, p.467-470, 1996.

OLIVEIRA, N.M.; OSÓRIO, J.C.; MONTEIRO, E.M. Produção de carne em ovinos de cinco genótipos. 4. Composição regional e tecidual. Ciência Rural, v.28, n.1, p.125-129, 1998.

OSÓRIO, J.C.; OLIVEIRA, N.M.; JARDIM, P.O. et al. Produção de carne em ovinos de cinco genótipos. 2. Componentes do peso vivo. Ciência Rural, v,26, n.3, p.471-475, 1996 a.
OSÓRIO, J.C.; OLIVEIRA, N.M.; NUNES, A.P. et al. Produção de carne em ovinos de cinco genótipos. 3. Perdas e morfologia. Ciência Rural, v.26, n.3, p.477-481, 1996b.

OSÓRIO, J.C.; AVILA, V.; JARDIM, P.O. et al. Produção de carne em cordeiros cruza Hampshire Down com Corriedale. Revista Brasileira Agrociência, v.2, n.2, p.99-104, 1996c.

OSÓRIO, J.C.; OSÓRIO, M.T.; JARDIM, P. et al. Métodos para avaliação de carne ovina: "in vivo", na carcaça e na carne. Pelotas: Universidade Federal de Pelotas, 1998a.107p.

OSÓRIO, J.C.; COSTA, J.C.; JARDIM, P. et al. Morfologia e características comerciais da produção de carne em cordeiros não castrados. 1. Efeito do genótipo. In: REUNIÃO ANUAL DA SOCIEDADE BRASILEIRA DE ZOOTECNIA, 35., 1998, Botucatu. Anais...Botucatu: Sociedade Brasileira de Zootecnia, 1998b. p.612-614.

OSÓRIO, J.C.; MARÍA, G.; JARDIM, P. et al. Caracteres de crecimiento, sacrificio y canal en corderos de raza Corriedale criados en un sistema sostenible sobre pastos naturales de Rio Grande do Sul (Brasil). Información Técnica Económica Agraria, v.94, n.1, p.63-73, 1998c.

OSÓRIO, J.C.; MARÍA, G.; BORBA, M. et al. Estudio comparativo de tres sistemas de producción de carne en ovinos Polwarth en Brasil. In: JORNADAS CIENTÍFICAS DE LA SOCIEDAD ESPAÑOLA DE OVINOTECNIA Y CAPRINOTECNIA, 23., 1998, Vitória-Gasteiz, España. Anais...Vitória-Gasteiz: SEOC, 1998d. p.461-464.

OSÓRIO, J.C.; SIERRA, I.; OLIVEIRA, N. et al. Estudio comparativo de tres sistemas de producción de carne en ovinos Corriedale en Brasil. In: JORNADAS CIENTÍFICAS DE LA SOCIEDAD ESPAÑOLA DE OVINOTECNIA Y CAPRINOTECNIA, 23., 1998. Vitória-Gasteiz, España. Anais... Vitória-Gasteiz: SEOC, 1998e. p.465-468.

OSÓRIO, J.C.; JARDIM, P.O.; PIMENTEL, M.A. et al. Produção de carne em cordeiros castrados e não castrados. 1 . Cruzas Hampshire Down x Corriedale. Ciência Rural, v.29, n.1, p.135-138, 1999a.

OSÓRIO, J.C.; MARÍA, G.; OLIVEIRA, N.M. et al. Estudio comparativo de tres sistemas de producción de carne en ovinos Polwarth. Revista Brasileira Agrociência, v.5, n.2, p.124-130, 1999b.

OSÓRIO, J.C.; OSÓRIO, M.T.; JARDIM, P. et al. Composição regional e tecidual em cordeiros Corriedale não castrados e castrados. In: REUNIÃO ANUAL DA SOCIEDADE BRASILEIRA DE ZOOTECNIA, 36., 1999, Porto Alegre. Anais...Porto Alegre: Sociedade Brasileira de Zootecnia, 1999c. CDROM. 4p.

OSÓRIO, M.T.; SIERRA, I.; SAÑUDO, C. et al. Influência da raça, sexo e peso/idade sobre o rendimento da carcaça em cordeiros. Ciência Rural, v.29, n.1, p.139-142, 1999 d.

OSÓRIO, J.C.; OSÓRIO, M.T.; FARIA, H. et al. Morfología “in vivo", en la canal y características productivas y comerciales en corderos Corriedale no castrados y castrados. In: JORNADAS CIENTÍFICAS DE LA SOCIEDAD ESPAÑOLA DE OVINOTECNIA Y CAPRINOTECNIA, 25., 2000. Teruel, España. Anais...Teruel: SEOC, 2000a, p.89-91.

OSÓRIO, J.C.; OLIVEIRA, N.M.; OSÓRIO, M.T., et al. Efecto de la edad al sacrificio sobre la producción de carne en corderos no castrados de cuatro razas. Revista Brasileira Agrociência, v.6, n.2, p.161-166, 2000b.

PALSSON, H.; VERGES, J.B. Effects of plane of nutrition on growth and development of carcass quality in lambs. Part I. The effects of haigh and low planes of nutrition at different ages. Journal of Agricultural Science, v.42, p.1-92, 1952a. 
PALSSON, H.; VERGES, J.B. Effects of plane of nutrition on growth and development os carcass quality in lambs. Part II. Effects on lambs of $30 \mathrm{lb}$ carcass weight. Journal of Agricultural Science, v.42, p.93-149, 1952b.

PIRES, C.C.; ARAÚJO, J.R.; BERNARDES, R.A. et al. Desempenho e características da carcaça de cordeiros de três grupos genéticos abatidos ao mesmo estágio de maturidade. Ciência Rural, v.29, n.1, p.155-158, 1999.

PIRES, C.C.; SILVA, L.F.; FARINATTI, L.H. et al. Crescimento de cordeiros abatidos com diferentes pesos. 2. Constituintes corporais. Ciência Rural, v.30, n.5, p.869-873, 2000.

ROQUE, A.P.; OSÓRIO, J.C.; JARDIM, P.O., et al. Produção de carne em ovinos de cinco genótipos. 6. Desenvolvimento relativo. Ciência Rural, v.29, n.3, p.549-553, 1999.

SAS INSTITUTE. Statistical Analysis System. User's Guide. 5.ed. Cary: 1985. 956p.
SIQUEIRA, E.R.; OSÓRIO, J.C.; GUERREIRO, J.L., et al Desempenho de cordeiros machos e fêmeas da raça Ideal e cruzas Texel x Ideal. Pesquisa Agropecuária Brasileira, v.19, n.12, p.1523-1528, 1984.

SIQUEIRA, E.R.; FERNANDES, S. Pesos, rendimentos e perdas da carcaça de cordeiros Corriedale e mestiços Ile de France x Corriedale, terminados em confinamento. Ciência Rural, v.29, n.1, p.143-148, 1999.

Recebido em: 26/07/01

Aceito em: 15/03/02 